\title{
Implementation and Evaluation of a Postpartum Care Program for Mothers Raising Infants: A Pilot Study
}

\author{
Kyoko Asazawa $\oplus^{\circledR}$, Natsuko Kojima, Tomoko Kato, Mieko Hirade \\ Division of Nursing, Faculty of Nursing, Tokyo Healthcare University, Tokyo, Japan \\ Email: 11DN001x2U-ts@slcn.ac.jp
}

How to cite this paper: Asazawa, K., Kojima, N., Kato, T. and Hirade, M. (2020) Implementation and Evaluation of a Postpartum Care Program for Mothers Raising Infants: A Pilot Study. Open Journal of Nursing, 10, 758-769.

https://doi.org/10.4236/ojn.2020.108054

Received: July 17, 2020

Accepted: August 17, 2020

Published: August 20, 2020

Copyright () 2020 by author(s) and Scientific Research Publishing Inc. This work is licensed under the Creative Commons Attribution International License (CC BY 4.0).

http://creativecommons.org/licenses/by/4.0/

\begin{abstract}
Background: The aims of this pilot study were to implement and evaluate a postpartum care program for mothers raising children younger than one year to provide physical relaxation, and to reduce mothers' parenting problems. Methods: A day-service and group-type postpartum care program was implemented with the aim of interacting with peers, promoting local communication, and relaxing mothers. Results: Forty-five pairs of mothers and infants participated in the study. Most of the mothers experienced relaxation, communicated with each other, resolved physical problems, reduced childcare concerns, and developed parenting friends. Conclusions: In addition, feasibility was assured as they were very satisfied with the content of programs such as baby massage and aroma treatment.
\end{abstract}

\section{Keywords}

Postnatal Care, Mothers, Midwifery, Program Evaluation, Feasibility Studies

\section{Introduction}

In Japan, the trend toward nuclear families, late marriages, older first-time mothers and declining birth rate has created a context of decreased sense of community and sparse interaction with children [1] [2]. Because women have few opportunities to interact with children in the community and often become mothers without acquiring child-rearing ability, child-rearing anxiety increases [3]. The situation is particularly prominent in the Tokyo metropolitan area, where, due to the aging parents who are mother supporters, new mothers must provide both elderly care and childcare [4].

Life devoted to child-rearing becomes a "closed room" for mothers and child- 
ren, making it difficult for them to have their own time, having few contact points with society, and being prone to obstructive and isolating situations [5]. In a systematic review by Gavin, Gaynes, Lohr, Meltzer-Brody, Gartlehner, \& Swinson, (2005) [6] postpartum depression was in the range of $6.5 \%$ to $12.9 \%$ of mothers within the first year of childbirth, especially those within three months of childbirth, with a prevalence of $19.2 \%$. Mothers who were prone to childcare anxiety and postpartum depression have been reported to be more likely to also abuse their children [7]. In a survey of mother's conditions after childbirth Shimada, et al. (2006) [8] found that for mothers with children under the age of one year: after giving birth had poorer physical and mental health such as lack of sleep, $(65.4 \%)$, problems with their breasts $(19.7 \%)$, loss of confidence in childcare, (12.5\%), and were feeling neglected (12.5\%).

There is over a decade of research about support for vulnerable postpartum mothers. Murphy et al. (2008) [9] found that in-home peer mentor support for first-time mothers in socio-disadvantages areas involved numerous logistical challenges for the peer mentors. These researchers concluded that more information about the potential difficulties and how support could resolve the difficulties is needed to optimize evaluation of the program [9]. Although in-home support provided by volunteer did not reduce the depression of postpartum mothers [10], telephone-based peer support was an effective strategy for preventing postpartum depression [11].

Shimada et al. (2006) [8] also surveyed the needs of new mothers. It became clear that there were strong needs for postpartum day care and childcare consultation by experts. Tanaka, Sakakibara, Kobayashi, \& Onodera, (2018) [12] found that it was important to support postpartum mothers with helpers who could listen therapeutically and has ample time to listen. Helpers needed to be approachable and locally available. Support is needed for local mothers to raise children with peace of mind, and in the care of women and families. In Japan, the importance of continuous care not only for pregnant women but also for postpartum mothers, newborns, and infants is being advocated [13]. On the other hand, in Taiwan and South Korea, care facilities and support systems for postpartum mothers are substantial [14]. In the UK, home-visit services after childbirth have been enhanced [15]. In Australia, mothers with infants have reduced childcare stress through online group-based nurse-led interventions [16]. In Japan, support systems for mothers and children are starting to be built.

The Ministry of Health, Labor and Welfare's, "Healthy Parents and Children 21 " has stated as basic issues health measures for uninterrupted care of pregnant women and infants and regional development to follow and nurture the healthy growth of children [1]. In addition, priority issues are supported close to parents who have difficulty raising children, and the need for a postpartum care business for local residents.

The academic institution's educational philosophy, to which the authors belong, is "a spirit that respects tolerance, warm humanity and respect for life" 
[17]. The Ministry of Education, Culture, Sports, Science and Technology (2003)

[18] recommended that the university fulfill its responsibilities, especially as a contribution to social development. Therefore, as part of establishing a postpartum care business, the authors needed to plan and implement a day service postpartum care program within their affiliated facility in order to contribute to childcare support for neighboring mothers and children. We decided to implement and evaluate a postpartum care program as a midwifery room at the University. The aims of this study were to implement and evaluate a postpartum care program for mothers raising children under the age of one to provide physical relaxation for the mother's, and to reduce mothers' parenting problems.

\section{Methods}

\subsection{Definition of Terms}

\subsubsection{Postpartum Care Program}

A series of programs for mothers and infants, including massages for infants, aroma hand treatments for mothers, breast-feeding consultations, childcare consultations, and discussion groups for mothers.

\subsubsection{Experienced Midwives}

Midwives who had worked for more than five years in midwifery clinic or hospitals and clinics that handle childbirth, and had provided many childcare and breastfeeding consultations for mothers and children.

\subsection{Design}

This was a quasi-experimental one-group posttest pilot study. The protocol for this study is shown in Figure 1.

\subsection{Participants and Recruitment}

This study was a study of program implementation and evaluation, and the main purpose of the study was to evaluate outcomes and process as a pilot study.

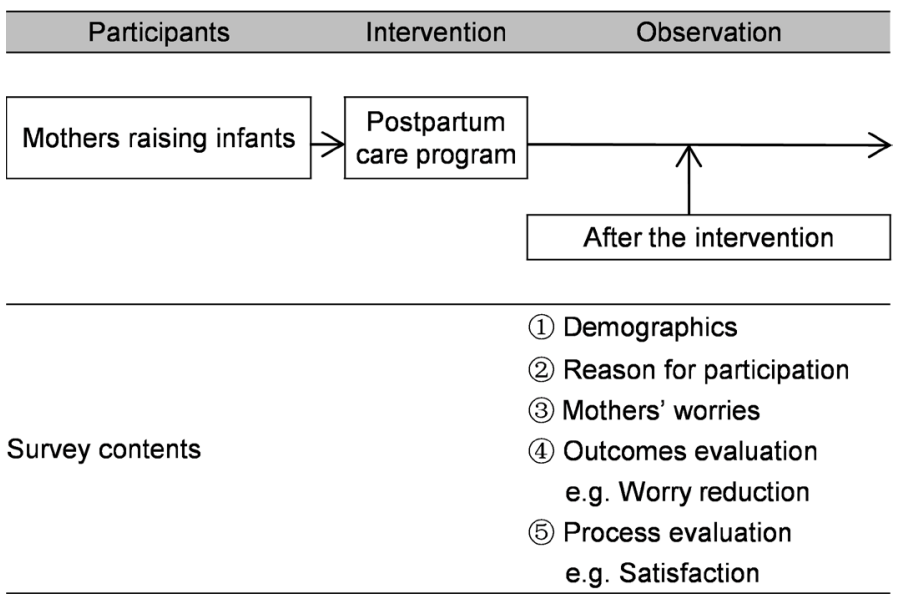

Figure 1. Intervention protocol of the present study. 
Therefore, 44 participants were calculated based on the number of participants expected to participate in the program among those held in a single year. To participate in the study, the following eligibility criteria had to be met: 1) a mother raising her infant and her infant, 2) living near the university, and 3) able to read and comprehend Japanese. Participants were recruited by posting posters in the waiting room of a nearby hospital, posting on the university website, and posting a poster at a nearby health center. The program was presented as a community-based approach located in a region of Tokyo Japan for local mothers and could contribute to making friends.

\subsection{Intervention}

A day-service and group-type postpartum care program was planned with the aim of peer interaction to strengthen social ties, promote local communication, and provide relaxation for mothers. It was planned with reference to relevant research on postpartum support [8]. The type of support that mothers wanted was outpatient consultation, and the contents of support were: advice for new lifestyle, childcare, breastfeeding, maternal rest and caring for self [19]. Therefore, it was determined that baby massage, a lactation consultation, and aroma treatment for mothers were appropriate for the program. The program contents are shown in Table 1. A round-table discussion was also set up for mothers to communicate with each other while enjoying tea and sweets. The baby massage was demonstrated and guided by a trained midwife. A midwife who was trained by a certified aromatherapy nurse provided the hand aroma treatment. A midwife specialized in lactation care provided consultation on breastfeeding. Experienced midwives staff served as a facilitator at the round-table discussions. Host midwives also performed childcare consultations such as baby food procedures, handling night crying, and skin moisturizing. The program was held once every two months. There were 6 - 12 participants per program. The direct intervention was 120 minutes (70 min group method, $50 \mathrm{~min}$ individual method). The implementation site was a Japanese-style room in the university, to which midwives who were also faculty belonged.

Table 1. Postpartum care program topics.

\begin{tabular}{ccc}
\hline Contents & Methods & Time (min) \\
Baby massage & Group & 30 \\
Aroma hand treatment & Individual & 15 \\
Breast-feeding consultation & Individual & 20 \\
Childcare consultation & Individual & 15 \\
Round-table discussion & Group & 40 \\
Total time & & 120
\end{tabular}

Note: ${ }^{*}$ mothers communicate with each other. 


\subsection{Measures}

The measurements were taken once immediately after the intervention. The face validity and content validity of the survey items were examined by four midwifery specialists with a master's degree in nursing or higher.

Demographics: Demographic information requested were: the number of children, the month ages of the infants, the ages of the mothers, the family type, worries during childcare, supporters, and the residential areas.

Reason for participation: Responses were requested regarding the number times they participated, participation motive, and purpose of participation.

Outcomes evaluation: Five items comprised the outcome assessment were investigated: interacting with peers, local communication promotion, mother's relaxation, solving postpartum physical questions, and reducing childcare worry. Each question was measured on a Likert scale from 1 (strongly disagree) to 5 (strongly agree); the higher the score, the higher participation's satisfaction.

Process evaluation: Total of eight items were investigated using yes/no response such as: satisfaction with the five program contents (baby massage, hand aroma treatment, breastfeeding consultation, childcare consultation, round-table discussions), match with expectation, appropriateness of time, and kindness of midwives. In addition, feedback on program participation was obtained using an open-ended format.

\subsection{Procedure}

To take into consideration the ethical aspects of participants, the research was carried out after obtaining approval from Tokyo Healthcare University Research Ethics Review Committee (30 - 45C). The program was run a total of eight times from April to December. We collected the measurements at the end of the program. The data collection period was from April to December, 2019. After explaining the purpose, procedure, method of this study, the participants voluntarily agreed to participate in the study. The researcher explained to participants about personal information protection clauses, protection of their benefits, ethical aspects of participation in the study, and there was no disadvantage for non-participation or dropout from the study. Each participant was asked to return the questionnaire in a sealed envelope by either mailing it or putting it in a collection box at the entrance of the university. Consequently, 47 pairs of mothers and infants participated and 45 valid responses (response-rate 95.7\%) were used for data analysis. Data with no missing values were selected in the collected questionnaires. The data were analyzed using the SPSS version 23.0 program. Statistics were obtained from the frequency distribution table of the obtained data.

\section{Results}

\subsection{Demographics}

A majority of participants were in their 30s; the age group of 30 to 34 years old 
was the highest with $37.8 \%$ (Table 2). Mothers with one child were $60.0 \%$ and $40.0 \%$ for two or more children. Infants were between 1 and 11 months of age, with $26.7 \%$ being the most common at 5 months of age. The majority of mothers (86.7\%) reported that husbands were their main childcare supporters. Mothers participated in the program because of baby massage (86.7\%), outing opportunities (64.4\%), and interest (46.7\%) (duplicate answers) (Figure 2). The worries of mothers were sleep deprivation (33.3\%), fatigue (31.1\%), procedure of baby foods (28.9\%), and trouble with breastfeeding (28.9\%) (duplicate answers) (see Figure 3).

Table 2. Participants demographic characteristics $(\mathrm{N}=45)$.

\begin{tabular}{|c|c|c|}
\hline Variable & $\mathrm{n}$ & $\%$ \\
\hline \multicolumn{3}{|l|}{ Age } \\
\hline $25-29$ & 9 & 20.0 \\
\hline $30-34$ & 17 & 37.8 \\
\hline $35-39$ & 15 & 33.3 \\
\hline over 40 & 4 & 8.9 \\
\hline \multicolumn{3}{|l|}{ Number of children } \\
\hline One child & 27 & 60.0 \\
\hline Two or more children & 18 & 40.0 \\
\hline \multicolumn{3}{|l|}{ Infants' age in month } \\
\hline One month & 1 & 2.2 \\
\hline Two months & 4 & 8.9 \\
\hline Three months & 5 & 11.1 \\
\hline Four months & 9 & 20.0 \\
\hline Five months & 12 & 26.7 \\
\hline Six months & 3 & 6.7 \\
\hline Seven months & 2 & 4.4 \\
\hline Eight months & 4 & 8.9 \\
\hline Nine months & 4 & 8.9 \\
\hline Eleven months & 1 & 2.2 \\
\hline \multicolumn{3}{|l|}{ The main support person } \\
\hline Husband & 39 & 86.7 \\
\hline Parents & 4 & 8.9 \\
\hline Friends & 2 & 4.4 \\
\hline
\end{tabular}




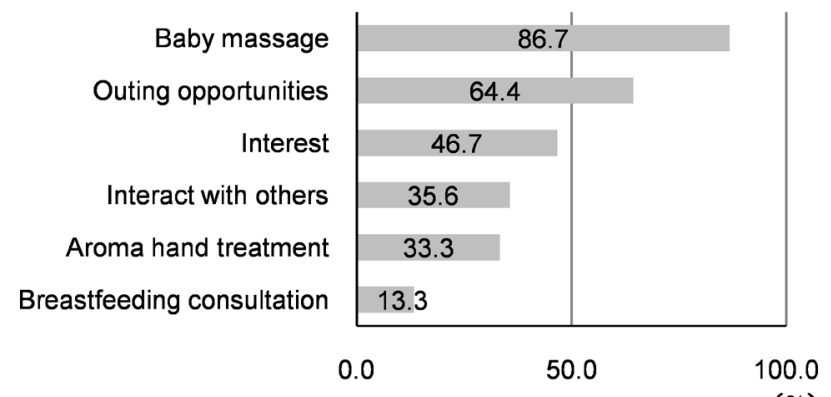

(\%)

Figure 2. Purpose of participation $(\mathrm{N}=45)$.

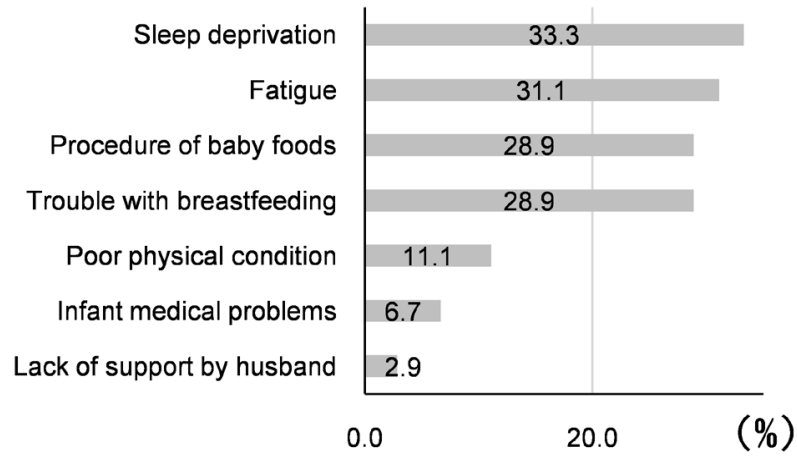

Figure 3. Mothers' worries $(\mathrm{N}=45)$.

\subsection{Outcome Evaluation}

In the outcome evaluation (see Figure 4), the vast majority (91.2\%) of the participants were satisfied with the increased communication and $100 \%$ felt that the program was a relaxation time. The mothers answered that the degree of achievement through the program were solving postpartum physical questions was $77.8 \%$, reducing childcare worries was $75.6 \%$, and interacting with peers was $71.1 \%$.

\subsection{Process Evaluation}

Satisfaction with program content (see Figure 5) was $97.8 \%$ for baby massage, 95.6\% for round-table discussions, $88.9 \%$ for aroma hand treatment, $77.8 \%$ for childcare consultation and $62.2 \%$ for breastfeeding consultation. The majority (71.1\%) of the participants indicated a high match between their expectations and the intervention, and $82.2 \%$ highly evaluated the provider's kindness.

\section{Discussion}

\subsection{Necessity of Postpartum Care Program}

The aim of this study was to implement and evaluate a postpartum care program for mothers raising infants. The provision of programs such as baby massage and aroma treatment was highly satisfactory. Baby massage was very satisfying because mothers enjoyed childcare and enjoyed the parent-child physical contact. A baby massage is effective in increasing the mother-infant attachment 


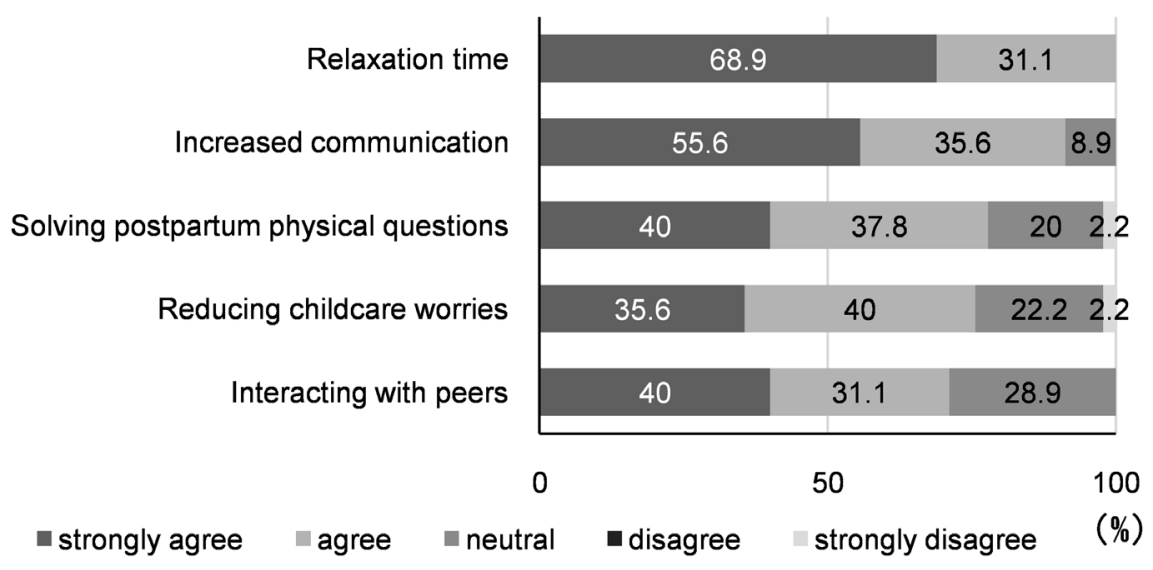

Figure 4. Outcome evaluation of the program $(\mathrm{N}=45)$.

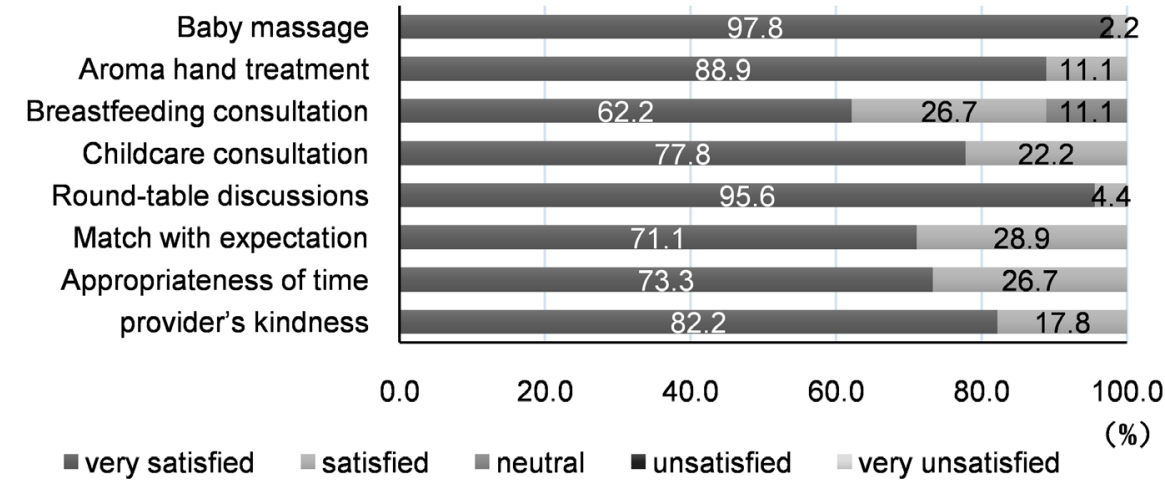

Figure 5. Satisfaction with program content $(\mathrm{N}=45)$.

[20]. The aroma treatment for mothers was highly satisfied with the care that was provided. According to previous studies, Aroma treatments were effective in improving relaxation and reducing fatigue for postpartum mothers [21]. It was presumed that because they were mothers immersed in childcare, they felt the value of care for themselves by others. Postnatal midwifery home care and connecting with midwives were found to be important in women's successful transition to motherhood in the early postnatal period [22]. Careful support by midwives is necessary for the mother's growth not only during hospitalization for delivery but also after returning to home after childbirth.

\subsection{Implications for Practice}

In addition, mothers became friends with each other through round-table discussions, and information on childcare was exchanged through conversations. This program promoted mothers making friends and contributed to peer support. This was a similar finding to the narrative systematic review that found peer support had a positive effect on postpartum mothers [23]. A qualitative study in England also found that peer supporters connected with and gave support to vulnerable and marginalized mothers, and enabled them to access services, in ways that complemented the work of health professionals [24]. Post- 
partum mothers receiving a peer support program tended to show better mental health outcomes [25]. Peer support is extremely useful, especially since new mothers are often lacking in childcare knowledge and experience.

The reason for the low level of satisfaction with breastfeeding consultations was that the infants were on average five months of age and breastfeeding was on track. The mothers also began to feed their infants with baby food, so it was considered that the interest in baby food was higher than breast milk. In order to improve the satisfaction of breastfeeding consultations, it is necessary to incorporate individual advice for each mother's breastfeeding status and information on when to stop breastfeeding. Care from midwives and communication with mothers who became friends in the program led to a high level of satisfaction and motivation for childcare. We would like to continue this program activity and contribute to the community.

\subsection{Strengths and Limitations}

This pilot study examined the feasibility of a program intervention in postpartum care by experienced midwives for mothers raising infants. The results show promise for providing relaxation and stress reduction for mothers. It is necessary to further analyze the outcome assessment using before-after analysis, and an intervention and control group. Moreover, the effectiveness of a postpartum care program needs to be further analyzed in a larger population and over time.

\section{Conclusion}

In this study, we evaluated the feasibility of a postpartum care program in order to provide relaxation for postpartum mothers both physically and mentally and to reduce mothers' childcare problems. We obtained the following important findings: Nearly all the participating mothers felt relaxed and satisfied with the communication with each other. Most mothers solved their physical problems, reduced childcare concerns, and developed friendships with other mothers. Many of the participants indicated a high satisfaction with the baby massage, their aroma hand treatment, and the round-table discussions. The program is feasible and should be considered for experimental research.

\section{Acknowledgements}

The authors would like to thank all the participants, as well as the facility staff and research assistants for their valuable contributions in this study.

\section{Disclosures}

Human rights statements and informed consent.

All procedures were performed in accordance with the ethical standards of the responsible committees for human experimentation (institutional and national) and the 1964 Helsinki Declaration and later amendments. Informed consent was obtained from all patients. 


\section{Animal Studies}

Animal studies were not performed in this study.

\section{Approval by Ethics Committee}

The protocol of this research project was approved by the Ethics Committee for Epidemiological Studies of Tokyo Healthcare University, Tokyo, Japan (approval no. 30 - 45C) on April 19, 2019.

\section{Conflicts of Interest}

The authors declare that there they have no conflicts of interest associated with this study.

\section{References}

[1] Ministry of Health, Labour and Welfare (2018) Healthy Parents and Children 21. http://sukoyaka21.jp/about2019.03.10

[2] Ogawa, K., Nakaoka, Y., Tomita, K., Maeda, K., Kato, K., Takahashi, J., et al. (2013) A Study on the Needs for Supporting Child Rearing in a Prefecture (Part 2): Factor Analysis of Child-Rearing Stresses. Bulletin of Shikoku University, 40, 13-19. (In Japanese) https://ci.nii.ac.jp/naid/120005847812

[3] Miyamoto, M., Funakoshi, K., Nakazoe, K., Tokioka, E., Mori, M. and Sibuya, S. (2000) Uneasiness of Mother for Child-Rearing and the Factors. Bulletin of Kagawa Prefectural College of Health Sciences, 2, 115-121. (In Japanese) https://ci.nii.ac.jp/naid/110000038428

[4] Muramatsu, N. and Akiyama, H. (2011) Japan: Super-Aging Society Preparing for the Future. Gerontologist, 51, 425-432. https://pubmed.ncbi.nlm.nih.gov/21804114 https://doi.org/10.1093/geront/gnr067

[5] Teshima, S. and Haraguchi, M. (2003) Childcare Support through Infant Health Checkups: Development of the Childcare Stress Scale. FPU Journal of Nursing Research, 1, 15-27. https://ci.nii.ac.jp/naid/120006367603

[6] Gavin, N.I., Gaynes, B.N., Lohr, K.N., Meltzer-Brody, S., Gartlehner, G. and Swinson, T. (2005) Perinatal Depression: A Systematic Review of Prevalence and Incidence. Obstetrics Gynecology, 106, 1071-1083.

https://www.ncbi.nlm.nih.gov/pubmed/16260528 https://doi.org/10.1097/01.AOG.0000183597.31630.db

[7] Mochizuki, M., Anme, T., Tanaka, E., Shinohara, R., Sugisawa, Y., Tomisaki, E., et al. (2014) The Influence of Caregivers' Anxiety and the Home Environment on Child Abuse: A Study of Children Attending Child-Care Centers. Japanese Journal of Public Health, 61, 263-273. (In Japanese) https://ci.nii.ac.jp/naid/130004684440

[8] Shimada, M, Sugimoto, M., Agata, T., Nitta, N., Seki, K., Ohashi, K., et al. (2006) Nationwide Survey on Maternal Anxiety in One-Month Postpartum, Needs for Childrearing Supports and Environment at Five Years after Healthy Parents and Children 21 Program. Journal of Child Health, 65, 752-762. (In Japanese) https://ci.nii.ac.jp/naid/10018584705

[9] Murphy, C.A., Cupples, M.E., Percy, A., Halliday, H.L. and Stewart, M.C. (2008) Peer-Mentoring for First-Time Mothers from Socio-Economic Disadvantage: A Qualitative Study within a Randomized Controlled Trial. BMC Health Services Research, 8, 46. https://pubmed.ncbi.nlm.nih.gov/18304334 
https://doi.org/10.1186/1472-6963-8-46

[10] Barnes, J., Senior, R. and MacPherson, K. (2009) The Utility of Volunteer Home-Visiting Support to Prevent Maternal Depression in the First Year of Life. Child Care, Health and Development, 35, 807-816. https://doi.org/10.1111/j.1365-2214.2009.01007.x

[11] Dennis, C.L. (2010) Postpartum Depression Peer Support: Maternal Perceptions from a Randomized Controlled Trial. International Journal of Nursing Studies, 47, 560-568. https://www.ncbi.nlm.nih.gov/pubmed/19962699 https://doi.org/10.1016/j.ijnurstu.2009.10.015

[12] Tanaka, M., Sakakibara, M., Kobayashi, Y. and Onodera, S. (2018) Conditions and Issues of Mother-Child Support at a Perinatal Care Center. The Bulletin of Health Science University, 14, 189-201. https://ci.nii.ac.jp/naid/130007432362

[13] Akashi, H., Ishioka, M., Hagiwara, A., Akashi, R. and Osanai, Y. (2018) Core Factors Promoting a Continuum of Care for Maternal, Newborn, and Child Health in Japan. Bioscience Trend, 12, 1-6. https://www.ncbi.nlm.nih.gov/pubmed/29479018 https://doi.org/10.5582/bst.2017.01304

[14] Sou, K. (2015) Postpartum Resting Period and Women's Body in Taiwan. Nara Women's University Sociological Studies, 22, 73-89. https://ci.nii.ac.jp/naid/120006657965

[15] Kamiya, S., Yamana, K., Ueno, H. and Matsuoka, E. (2015) The Actual Maternity Care System and Birth Center of the National Health Service in the UK. Bulletin of Aichi Prefectural University School of Nursing and Health, 21, 89-97. https://ci.nii.ac.jp/naid/120005719316/amp/en

[16] Sawyer, A., Kaim, A., Le, H.N., McDonald, D., Mittinty, M., Lynch, J. and Sawyer, M. (2019) The Effectiveness of an App-Based Nurse-Moderated Program for New Mothers with Depression and Parenting Problems (eMums Plus): Pragmatic Randomized Controlled Trial. Journal Medical Internet Research, 21, e13689. https://doi.org/10.2196/13689

[17] Tokyo Healthcare University (2019) About Tokyo Healthcare University. http://www.thcu.ac.jp/about/idea.html

[18] Ministry of Education, Culture, Sports, Science and Technology (2003) Significance of Industry-Academia-Government Collaboration. https://www.mext.go.jp/b_menu/shingi/gijyutu/gijyutu8/toushin/attach/1332039.htm

[19] Sakanashi, K., Katsukawa, Y., Mizuno, S., Usui, M. and Nabeta, M. (2014) Support Desired by Mothers Following Postpartum Hospital Discharge: Based on the Preferences of Mothers with Infants Less than 4 Months Old. Kanto Gakuin University Journal of Nursing, 1, 16-24. https://ci.nii.ac.jp/naid/120006025929

[20] Gürol, A. and Polat, S. (2012) The Effects of Baby Massage on Attachment between Mother and their Infants. Asian Nursing Research, 6, 35-41.

https://pubmed.ncbi.nlm.nih.gov/25030689 https://doi.org/10.1016/j.anr.2012.02.006

[21] Asazawa, K., Kato, Y., Yamaguchi, A. and Inoue, A. (2017) The Effect of Aromatherapy Treatment on Fatigue and Relaxation for Mothers during the Early Puerperal Period in Japan: A Pilot Study. International Journal of Community Based Nursing \& Midwifery, 5, 365-375. https://www.ncbi.nlm.nih.gov/pubmed/29043282

[22] Walker, S.B., Rossi, D.M. and Sander, T.M. (2019) Women's Successful Transition to Motherhood during the Early Postnatal Period: A Qualitative Systematic Review of Postnatal and Midwifery Home Care Literature. Midwifery, 79, Article ID: 102552. https://pubmed.ncbi.nlm.nih.gov/31605940 
https://doi.org/10.1016/j.midw.2019.102552

[23] Leger, J. and Letourneau, N. (2015) New Mothers and Postpartum Depression: A Narrative Review of Peer Support Intervention Studies. Health Social Care Community, 23, 337-348. https://pubmed.ncbi.nlm.nih.gov/25346377 https://doi.org/10.1111/hsc. 12125

[24] McLeish, J. and Redshaw, M. (2015) Peer Support during Pregnancy and Early Parenthood: A Qualitative Study of Models and Perceptions. BMC Pregnancy Childbirth, 15, 257. https://pubmed.ncbi.nlm.nih.gov/26459281 https://doi.org/10.1186/s12884-015-0685-y

[25] Shorey, S., Chee, C.Y.I., Ng, E.D., Lau, Y., Dennis, C.L. and Chan, Y.H. (2019) Evaluation of a Technology-Based Peer-Support Intervention Program for Preventing Postnatal Depression (Part 1): Randomized Controlled Trial. Journal Medical Internet Research, 21, e12410. https://pubmed.ncbi.nlm.nih.gov/31469084

https://doi.org/10.2196/12410 